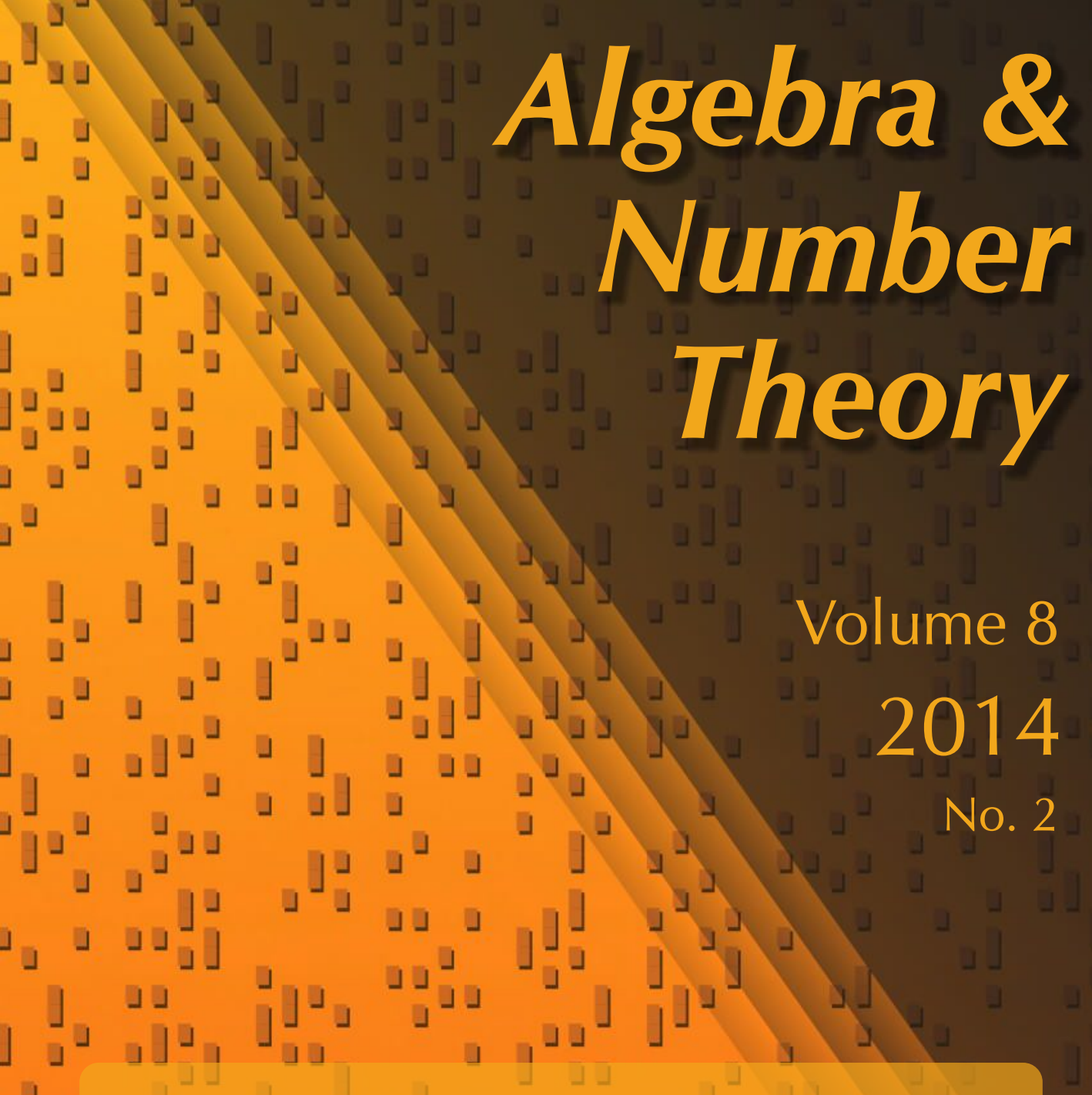

Algebraicity of the zeta function associated to a matrix over a free group algebra

Christian Kassel and Christophe Reutenauer

\lrcorner .

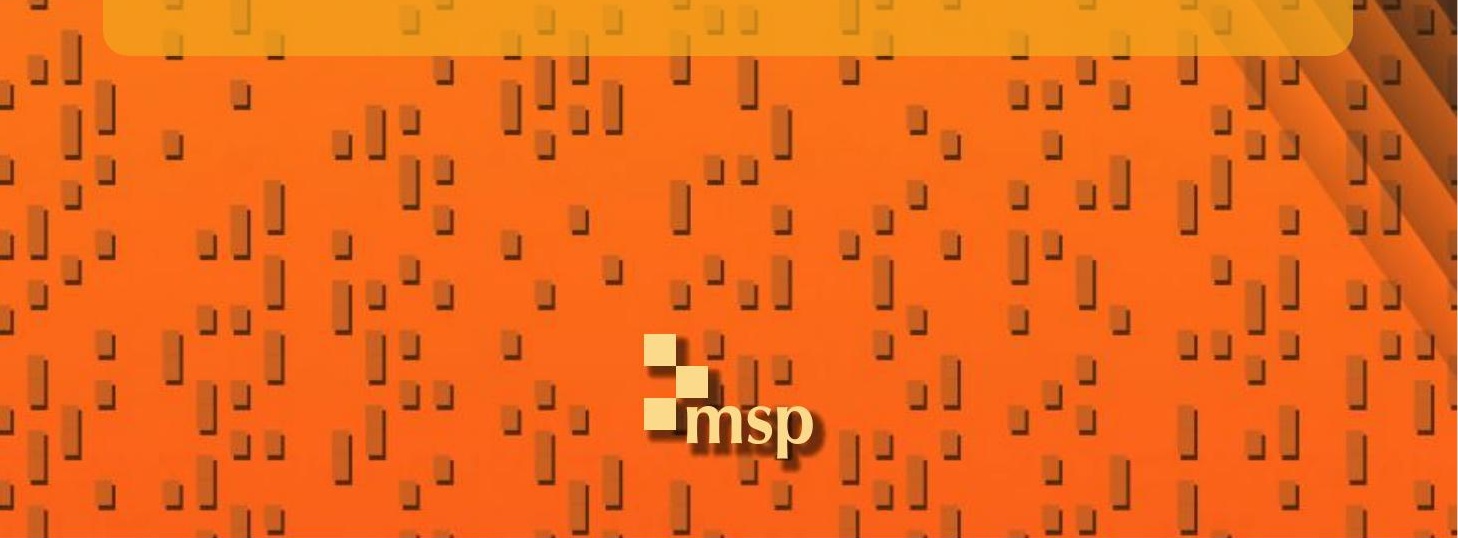




\title{
Algebraicity of the zeta function associated to a matrix over a free group algebra
}

\author{
Christian Kassel and Christophe Reutenauer
}

Following and generalizing a construction by Kontsevich, we associate a zeta function to any matrix with entries in a ring of noncommutative Laurent polynomials with integer coefficients. We show that such a zeta function is an algebraic function.

\section{Introduction}

Fix a commutative ring $K$. Let $F$ be a free group on a finite number of generators $X_{1}, \ldots, X_{n}$ and

$$
K F=K\left\langle X_{1}, X_{1}^{-1}, \ldots, X_{n}, X_{n}^{-1}\right\rangle
$$

be the corresponding group algebra: equivalently, it is the algebra of noncommutative Laurent polynomials with coefficients in $K$. Any element $a \in K F$ can be uniquely written as a finite sum of the form

$$
a=\sum_{g \in F}(a, g) g
$$

where $(a, g) \in K$.

Let $M$ be a $d \times d$ matrix with coefficients in $K F$. For any $n \geq 1$, we may consider the $n$-th power $M^{n}$ of $M$ and its trace $\operatorname{Tr}\left(M^{n}\right)$, which is an element of $K F$. We define the integer $a_{n}(M)$ as the coefficient of 1 in the trace of $M^{n}$ :

$$
a_{n}(M)=\left(\operatorname{Tr}\left(M^{n}\right), 1\right) .
$$

Let $g_{M}$ and $P_{M}$ be the formal power series

$$
g_{M}=\sum_{n \geq 1} a_{n}(M) t^{n} \quad \text { and } \quad P_{M}=\exp \left(\sum_{n \geq 1} a_{n}(M) \frac{t^{n}}{n}\right) .
$$

They are related by

$$
g_{M}=t \frac{d \log \left(P_{M}\right)}{d t} .
$$

MSC2010: primary 05A15, 68Q70, 68R15; secondary 05E15, 14H05, $14 \mathrm{G} 10$.

Keywords: noncommutative formal power series, language, zeta function, algebraic function. 
We call $P_{M}$ the zeta function of the matrix $M$ by analogy with the zeta function of a noncommutative formal power series (see next section); the two concepts will be related in Proposition 4.1.

The motivation for the definition of $P_{M}$ comes from the well-known identity expressing the inverse of the reciprocal polynomial of the characteristic polynomial of a matrix $M$ with entries in a commutative ring

$$
\frac{1}{\operatorname{det}(1-t M)}=\exp \left(\sum_{n \geq 1} \operatorname{Tr}\left(M^{n}\right) \frac{t^{n}}{n}\right) .
$$

Note that, for any scalar $\lambda \in K$, the corresponding series for the matrix $\lambda M$ become

$$
g_{\lambda M}(t)=g_{M}(\lambda t) \quad \text { and } \quad P_{\lambda M}(t)=P_{M}(\lambda t) .
$$

Our main result is the following; it was inspired by Theorem 1 of [Kontsevich 2011]:

Theorem 1.1. For each matrix $M \in M_{d}(K F)$ where $K=\mathbb{Q}$ is the ring of rational numbers, the formal power series $P_{M}$ is algebraic.

The special case $d=1$ is due to Kontsevich [2011]. A combinatorial proof in the case $d=1$ and $F$ is a free group on one generator appears in [Reutenauer and Robado 2012].

Observe that by the rescaling equalities (1-3) it suffices to prove the theorem when $K=\mathbb{Z}$ is the ring of integers.

It is crucial for the veracity of Theorem 1.1 that the variables do not commute: for instance, if $a=x+y+x^{-1}+y^{-1} \in \mathbb{Z}\left[x, x^{-1}, y, y^{-1}\right]$, where $x$ and $y$ are commuting variables, then $\exp \left(\sum_{n \geq 1}\left(a^{n}, 1\right) t^{n} / n\right)$ is a formal power series with integer coefficients but not an algebraic function (this follows from Example 3 in [Bousquet-Mélou 2005, §1]).

The paper is organized as follows. In Section 2, we define the zeta function $\zeta_{S}$ of a noncommutative formal power series $S$ and show that it can be expanded as an infinite product under a cyclicity condition that is satisfied by the characteristic series of cyclic languages.

In Section 3, we recall the notion of algebraic noncommutative formal power series and some of their properties.

In Section 4, we reformulate the zeta function of a matrix as the zeta function of a noncommutative formal power series before giving the proof of Theorem 1.1; the latter follows the steps sketched in [Kontsevich 2011] and relies on the results of the previous sections as well as on an algebraicity result by André [2004] elaborating on an idea of D. and G. Chudnovsky.

We concentrate on two specific matrices in Section 5. We give a closed formula for the zeta function of the first matrix; its nonzero coefficients count the planar 
rooted bicubic maps as well as Chapoton's "new intervals" in a Tamari lattice (see [Chapoton 2006; Tutte 1963]).

\section{Cyclic formal power series}

General definitions. As usual, if $A$ is a set, we denote by $A^{*}$ the free monoid on $A$ : it consists of all words on the alphabet $A$, including the empty word 1 .

Let $A^{+}=A-\{1\}$. Recall that $w \in A^{+}$is primitive if it cannot be written as $u^{r}$ for any integer $r \geq 2$ and any $u \in A^{+}$. Two elements $w, w^{\prime} \in A^{+}$are conjugate if $w=u v$ and $w^{\prime}=v u$ for some $u, v \in A^{*}$.

Given a set $A$ and a commutative ring $K$, let $K\langle\langle A\rangle$ be the algebra of noncommutative formal power series on the alphabet $A$. For any element $S \in K\langle\langle A\rangle\rangle$ and any $w \in A^{*}$, we define the coefficient $(S, w) \in K$ by

$$
S=\sum_{w \in A^{*}}(S, w) w .
$$

As an example of such noncommutative formal power series, take the characteristic series $\sum_{w \in L} w$ of a language $L \subseteq A^{*}$. In the sequel, we shall identify a language with its characteristic series.

The generating series $g_{S}$ of an element $S \in K\langle\langle A\rangle\rangle$ is the image of $S$ under the algebra map $\varepsilon: K\langle\langle A\rangle \rightarrow K \llbracket t \rrbracket$ sending each $a \in A$ to the variable $t$. We have

$$
g_{S}-(S, 1)=\sum_{w \in A^{+}}(S, w) t^{|w|}=\sum_{n \geq 1}\left(\sum_{|w|=n}(S, w)\right) t^{n},
$$

where $|w|$ is the length of $w$.

The zeta function $\zeta_{S}$ of $S \in K\langle\langle A\rangle\rangle$ is defined by

$$
\zeta_{S}=\exp \left(\sum_{w \in A^{+}}(S, w) \frac{t^{|w|}}{|w|}\right)=\exp \left(\sum_{n \geq 1}\left(\sum_{|w|=n}(S, w)\right) \frac{t^{n}}{n}\right) .
$$

The formal power series $g_{S}$ and $\zeta_{S}$ are related by

$$
t \frac{d \log \left(\zeta_{S}\right)}{d t}=t \frac{\zeta_{S}^{\prime}}{\zeta_{S}}=g_{S}-(S, 1)
$$

where $\zeta_{S}^{\prime}$ is the derivative of $\zeta_{S}$ with respect to the variable $t$.

\section{Cyclicity.}

Definition 2.1. An element $S \in K\langle\langle A\rangle\rangle$ is cyclic if

(i) $\forall u, v \in A^{*},(S, u v)=(S, v u)$ and

(ii) $\forall w \in A^{+}, \forall r \geq 2,\left(S, w^{r}\right)=(S, w)^{r}$. 
Cyclic languages provide examples of cyclic formal power series. Recall from [Berstel and Reutenauer 1990, §2] that a language $L \subseteq A^{*}$ is cyclic if

(1) $\forall u, v \in A^{*}, u v \in L \Longleftrightarrow v u \in L$ and

(2) $\forall w \in A^{+}, \forall r \geq 2, \quad w^{r} \in L \Longleftrightarrow w \in L$.

The characteristic series of a cyclic language is a cyclic formal power series in the above sense.

Let $L$ be any set of representatives of conjugacy classes of primitive elements of $A^{+}$.

Proposition 2.2. If $S \in K\langle\langle A\rangle\rangle$ is a cyclic formal power series, then

$$
\zeta_{S}=\prod_{\ell \in L} \frac{1}{1-(S, \ell) t^{|\ell|}} .
$$

Proof. Since both sides of the equation have the same constant term 1, it suffices to prove that they have the same logarithmic derivative. The logarithmic derivative of the right-hand side multiplied by $t$ is equal to

$$
\sum_{\ell \in L} \frac{|\ell|(S, \ell) t^{|\ell|}}{1-(S, \ell) t^{|\ell|}},
$$

which in turn is equal to

$$
\sum_{\ell \in L, k \geq 1}|\ell|(S, \ell)^{k} t^{k|\ell|}
$$

In view of (2-1) and (2-3), it is enough to check that, for all $n \geq 1$,

$$
\sum_{|w|=n}(S, w)=\sum_{\ell \in L, k \geq 1, k|\ell|=n}|\ell|(S, \ell)^{k}
$$

Now any word $w=u^{k}$ is the $k$-th power of a unique primitive word $u$, which is the conjugate of a unique element $\ell \in L$. Moreover, $w$ has exactly $|\ell|$ conjugates and, since $S$ is cyclic, we have

$$
(S, w)=\left(S, u^{k}\right)=(S, u)^{k}=(S, \ell)^{k} .
$$

From this, Equation (2-4) follows immediately.

Corollary 2.3. If a cyclic formal power series $S$ has integer coefficients, that is, if $(S, w) \in \mathbb{Z}$ for all $w \in A^{*}$, then so does $\zeta_{S}$. 


\section{Algebraic noncommutative series}

This section is essentially a compilation of well-known results on algebraic noncommutative series.

Recall that a system of proper algebraic noncommutative equations is a finite set of equations

$$
\xi_{i}=p_{i}, \quad i=1, \ldots, n,
$$

where $\xi_{1}, \ldots, \xi_{n}$ are noncommutative variables and $p_{1}, \ldots, p_{n}$ are elements of $K\left\langle\xi_{1}, \ldots, \xi_{n}, A\right\rangle$, where $A$ is some alphabet. We assume that each $p_{i}$ has no constant term and contains no monomial $\xi_{j}$. One can show that such a system has a unique solution $\left(S_{1}, \ldots, S_{n}\right)$, i.e., there exists a unique $n$-tuple $\left(S_{1}, \ldots, S_{n}\right) \in$ $K\langle\langle A\rangle\rangle^{n}$ such that $S_{i}=p_{i}\left(S_{1}, \ldots, S_{n}, A\right)$ for all $i=1, \ldots, n$ and each $S_{i}$ has no constant term (see [Schützenberger 1962], [Salomaa and Soittola 1978, Theorem IV.1.1], or [Stanley 1999, Proposition 6.6.3]).

If a formal power series $S \in K\langle\langle A\rangle$ differs by a constant from such a formal power series $S_{i}$, we say that $S$ is algebraic.

Example 3.1. Consider the proper algebraic noncommutative equation

$$
\xi=a \xi^{2}+b .
$$

(Here $A=\{a, b\}$.) Its solution is of the form

$$
S=b+a b b+a a b b b+a b a b b+\cdots .
$$

One can show (see [Berstel 1979]) that $S$ is the characteristic series of Łukasiewicz's language, namely of the set of words $w \in\{a, b\}^{*}$ such that $|w|_{b}=|w|_{a}+1$ and $|u|_{a} \geq|u|_{b}$ for all proper prefixes $u$ of $w$.

Recall also that $S \in K\langle\langle A\rangle\rangle$ is rational if it belongs to the smallest subalgebra of $K\langle\langle A\rangle\rangle$ containing $K\langle A\rangle$ and closed under inversion. By a theorem of Schützenberger (see [Berstel and Reutenauer 2011, Theorem I.7.1]), a formal power series $S \in K\langle\langle A\rangle\rangle$ is rational if and only if it is recognizable, i.e., there exist an integer $n \geq 1$, a representation $\mu$ of the free monoid $A^{*}$ by matrices with entries in $K$, a row-matrix $\alpha$ and a column-matrix $\beta$ such that, for all $w \in A^{*}$,

$$
(S, w)=\alpha \mu(w) \beta .
$$

We now record two well-known theorems.

Theorem 3.2. (1) If $S \in K\left\langle\langle A\rangle\right.$ is algebraic, then its generating series $g_{S} \in K \llbracket t \rrbracket$ is algebraic in the usual sense.

(2) The set of algebraic power series is a subring of $K\langle\langle A\rangle$.

(3) A rational power series is algebraic. 
(4) The Hadamard product of a rational power series and an algebraic power series is algebraic.

(5) Let $A=\left\{a_{1}, \ldots, a_{n}, a_{1}^{-1}, \ldots, a_{n}^{-1}\right\}$ and $L$ be the language consisting of all words on the alphabet $A$ whose image in the free group on $a_{1}, \ldots, a_{n}$ is the neutral element. Then the characteristic series of $L$ is algebraic.

Items (1)-(4) of the previous theorem are due to Schützenberger [1962] and Item (5) to Chomsky and Schützenberger [1963] (see [Stanley 1999, Example 6.6.8]).

The second theorem is a criterion due to Jacob [1975].

Theorem 3.3. A formal power series $S \in K\langle\langle A\rangle\rangle$ is algebraic if and only if there exist a free group $F$, a representation $\mu$ of the free monoid $A^{*}$ by matrices with entries in $K F$, indices $i$ and $j$, and an element $\gamma \in F$ such that, for all $w \in A^{*}$,

$$
(S, w)=\left((\mu w)_{i, j}, \gamma\right)
$$

The following is an immediate consequence of Theorem 3.3:

Corollary 3.4. If $S \in K\left\langle\langle A\rangle\right.$ is an algebraic power series and $\varphi: B^{*} \rightarrow A^{*}$ is a homomorphism of finitely generated free monoids, then the power series

$$
\sum_{w \in B^{*}}(S, \varphi(w)) w \in K\langle\langle B\rangle\rangle
$$

is algebraic.

As a consequence of Theorem 3.2(5) and of Corollary 3.4, we obtain:

Corollary 3.5. Let $f: A^{*} \rightarrow F$ be a homomorphism from $A^{*}$ to a free group $F$. Then the characteristic series of $f^{-1}(1) \in K\langle\langle A\rangle$ is algebraic.

\section{Proof of Theorem 1.1}

Let $M$ be a $d \times d$ matrix. As observed in the introduction, it is enough to establish Theorem 1.1 when all the entries of $M$ belong to $\mathbb{Z} F$.

We first reformulate the formal power series $g_{M}$ and $P_{M}$ of (1-2) as the generating series and the zeta function of a noncommutative formal power series, respectively.

Let $A$ be the alphabet whose elements are triples $[g, i, j]$, where $i$ and $j$ are integers such that $1 \leq i, j \leq d$ and $g \in F$ appears in the $(i, j)$-entry $M_{i, j}$ of $M$, i.e., $\left(M_{i, j}, g\right) \neq 0$. We define the noncommutative formal power series $S_{M} \in K\langle\langle A\rangle\rangle$ as follows: for $w=\left[g_{1}, i_{1}, j_{1}\right] \cdots\left[g_{n}, i_{n}, j_{n}\right] \in A^{+}$, the scalar $\left(S_{M}, w\right)$ vanishes unless we have

(a) $j_{n}=i_{1}$ and $j_{k}=i_{k+1}$ for all $k=1, \ldots, n-1$ and

(b) $g_{1} \cdots g_{n}=1$ in the group $F$, 
in which case $\left(S_{M}, w\right)$ is given by

$$
\left(S_{M}, w\right)=\left(M_{i_{1}, j_{1}}, g_{1}\right) \cdots\left(M_{i_{n}, j_{n}}, g_{n}\right) \in K .
$$

By convention, $\left(S_{M}, 1\right)=d$.

Proposition 4.1. The generating series and the zeta function of $S_{M}$ are related to the formal power series $g_{M}$ and $P_{M}$ of (1-2) by

$$
g_{S_{M}}-d=g_{M} \text { and } \zeta_{S_{M}}=P_{M} .
$$

Proof. For $n \geq 1$, we have

$$
\begin{aligned}
\operatorname{Tr}\left(M^{n}\right) & =\sum M_{i_{1}, j_{1}} \cdots M_{i_{n}, j_{n}} \\
& =\sum\left(M_{i_{1}, j_{1}}, g_{1}\right) \cdots\left(M_{i_{n}, j_{n}}, g_{n}\right) g_{1} \cdots g_{n},
\end{aligned}
$$

where the sum runs over all indices $i_{1}, j_{1}, \ldots, i_{n}, j_{n}$ satisfying Condition (a) above and over all $g_{1}, \ldots, g_{n} \in F$. Then

$$
a_{n}(M)=\left(\operatorname{Tr}\left(M^{n}\right), 1\right)=\sum\left(M_{i_{1}, j_{1}}, g_{1}\right) \cdots\left(M_{i_{n}, j_{n}}, g_{n}\right),
$$

where Conditions (a) and (b) are satisfied. Hence,

$$
a_{n}(M)=\sum_{w \in A^{*},|w|=n}(S, w),
$$

which proves the proposition in view of (1-2), (2-1) and (2-2).

We next establish that $S_{M}$ is both cyclic in the sense of Section 2 and algebraic in the sense of Section 3.

Proposition 4.2. The noncommutative formal power series $S_{M}$ is cyclic.

Proof. (i) Conditions (a) and (b) above are clearly preserved under cyclic permutations. Hence, we also have

$$
\left(S_{M}, w\right)=\left(M_{i_{2}, j_{2}}, g_{2}\right) \cdots\left(M_{i_{n}, j_{n}}, g_{n}\right)\left(M_{i_{1}, j_{1}}, g_{1}\right)
$$

when $w=\left[g_{1}, i_{1}, j_{1}\right] \cdots\left[g_{n}, i_{n}, j_{n}\right]$ such that Conditions (a) and (b) are satisfied. It follows that $(S, u v)=S(v u)$ for all $u, v \in A^{*}$.

(ii) If $w$ satisfies Conditions (a) and (b), so does $w^{r}$ for $r \geq 2$. Conversely, if $w^{r}$ $(r \geq 2)$ satisfies Condition (a), then since

$$
w^{r}=\left[g_{1}, i_{1}, j_{1}\right] \cdots\left[g_{n}, i_{n}, j_{n}\right]\left[g_{1}, i_{1}, j_{1}\right] \cdots
$$

we must have $j_{n}=i_{1}$ and $j_{k}=i_{k+1}$ for all $k=1, \ldots, n-1$, and so $w$ satisfies Condition (a).

If $w^{r}(r \geq 2)$ satisfies Condition (b), i.e., $\left(g_{1} \cdots g_{n}\right)^{r}=1$, then $g_{1} \cdots g_{n}=1$ since $F$ is torsion-free. Hence, $w$ satisfies Condition (b). It follows that $\left(S, w^{r}\right)=$ $\left(\left(M_{i_{1}, j_{1}}, g_{1}\right) \cdots\left(M_{i_{n}, j_{n}}, g_{n}\right)\right)^{r}=(S, w)^{r}$. 
Proposition 4.3. The noncommutative formal power series $S_{M}$ is algebraic.

Proof. We write $S_{M}$ as the Hadamard product of three noncommutative formal power series $S_{1}, S_{2}$ and $S_{3}$.

The series $S_{1} \in K\langle\langle A\rangle\rangle$ is defined for $w=\left[g_{1}, i_{1}, j_{1}\right] \cdots\left[g_{n}, i_{n}, j_{n}\right] \in A^{+}$by

$$
\left(S_{1}, w\right)=\left(M_{i_{1}, j_{1}}, g_{1}\right) \cdots\left(M_{i_{n}, j_{n}}, g_{n}\right)
$$

and by $\left(S_{1}, 1\right)=1$. This is a recognizable, hence rational, series with onedimensional representation $A^{*} \rightarrow K$ given by $[g, i, j] \mapsto\left(M_{i, j}, g\right)$.

Next consider the representation $\mu$ of the free monoid $A^{*}$ defined by

$$
\mu([g, i, j])=E_{i, j},
$$

where $E_{i, j}$ denotes as usual the $d \times d$ matrix with all entries vanishing except the $(i, j)$-entry, which is equal to 1 . Set

$$
S_{2}=\sum_{w \in A^{*}} \operatorname{Tr}((\mu w)) w \in K\langle\langle A\rangle\rangle .
$$

The power series $S_{2}$ is recognizable and hence rational. Let us describe $S_{2}$ more explicitly. For $w=1, \mu(w)$ is the identity $d \times d$ matrix; hence, $\left(S_{2}, 1\right)=d$. For $w=\left[g_{1}, i_{1}, j_{1}\right] \cdots\left[g_{n}, i_{n}, j_{n}\right] \in A^{+}$, we have

$$
\operatorname{Tr}((\mu w))=\operatorname{Tr}\left(E_{i_{1}, j_{1}} \cdots E_{i_{n}, j_{n}}\right) .
$$

It follows that $\operatorname{Tr}((\mu w)) \neq 0$ if and only if $\operatorname{Tr}\left(E_{i_{1}, j_{1}} \cdots E_{i_{n}, j_{n}}\right) \neq 0$, which is equivalent to $j_{n}=i_{1}$ and $j_{k}=i_{k+1}$ for all $k=1, \ldots, n-1$, in which case $\operatorname{Tr}((\mu w))=1$. Thus,

$$
S_{2}=d+\sum_{n \geq 1} \sum\left[g_{1}, i_{1}, i_{2}\right]\left[g_{2}, i_{2}, i_{3}\right] \cdots\left[g_{n}, i_{n}, i_{1}\right],
$$

where the second sum runs over all elements $g_{1}, \ldots, g_{n} \in F$ and all indices $i_{1}, \ldots, i_{n}$.

Finally, consider the homomorphism $f: A^{*} \rightarrow F$ sending $[g, i, j]$ to $g$. Then by Corollary 3.5 the characteristic series $S_{3} \in K\left\langle\langle A\rangle\right.$ of $f^{-1}(1)$ is algebraic.

It is now clear that $S_{M}$ is the Hadamard product of $S_{1}, S_{2}$ and $S_{3}$ :

$$
S_{M}=S_{1} \odot S_{2} \odot S_{3} .
$$

Since, by [Berstel and Reutenauer 2011, Theorem I.5.5] the Hadamard product of two rational series is rational, $S_{1} \odot S_{2}$ is rational as well. It then follows from Theorem 3.2(4) and the algebraicity of $S_{3}$ that $S_{M}=S_{1} \odot S_{2} \odot S_{3}$ is algebraic.

Since $M$ has entries in $\mathbb{Z} F$, the power series $g_{S_{M}}=g_{M}+d$ belongs to $\mathbb{Z} \llbracket t \rrbracket$. It follows by Corollary 2.3 and Proposition 4.2 that the power series $P_{M}=\zeta_{S_{M}}$ has 
integer coefficients as well. Moreover, by Theorem 3.2(1) and Proposition 4.3,

$$
t \frac{d \log \left(P_{M}\right)}{d t}=g_{M}
$$

is algebraic.

To complete the proof of Theorem 1.1, it suffices to apply the following algebraicity theorem:

Theorem 4.4. If $f \in \mathbb{Z} \llbracket t \rrbracket$ is a formal power series with integer coefficients such that $t d \log f / d t$ is algebraic, then $f$ is algebraic.

Note that the integrality condition for $f$ is essential: for the transcendental formal power series $f=\exp (t)$, we have $t d \log f / d t=t$, which is even rational.

Proof. This result follows from cases of the Grothendieck-Katz conjecture proved in [André 2004] and in [Bost 2001]. The conjecture states that, if $Y^{\prime}=A Y$ is a linear system of differential equations with $A \in M_{d}(\mathbb{Q}(t))$, then far from the poles of $A$ it has a basis of solutions that are algebraic over $\mathbb{Q}(t)$ if and only if for almost all prime numbers $p$ the reduction $\bmod p$ of the system has a basis of solutions that are algebraic over $\mathbb{F}_{p}(t)$.

Let us now sketch a proof of the theorem (see also Exercise 5 of [André 1989, p. 160]). Set $g=t f^{\prime} / f$, and consider the system $y^{\prime}=(g / t) y$; it defines a differential form $\omega$ on an open set $S$ of the smooth projective complete curve $\bar{S}$ associated to $g$. We now follow [André 2004, §6.3], which is inspired from [Chudnovsky and Chudnovsky 1985]. First, extend $\omega$ to a section (still denoted $\omega$ ) of $\Omega_{\bar{S}}^{1}(-D)$, where $D$ is the divisor of poles of $\omega$. For any $n \geq 2$, we have a differential form $\sum_{i=1}^{n} p_{i}^{*}(\omega)$ on $S^{n}$, where $p_{i}: S^{n} \rightarrow S$ is the $i$-th canonical projection; this form goes down to the symmetric power $S^{(n)}$. Now let $J$ be the generalized Jacobian of $S$ parametrizing invertible fiber bundles over $\bar{S}$ that are rigidified over $D$. There is a morphism $\varphi: S \rightarrow J$ and a unique invariant differential form $\omega_{J}$ on $J$ such that $\omega=\varphi^{*}\left(\omega_{J}\right)$. For any $n \geq 2, \varphi$ induces a morphism $\varphi^{(n)}: S^{(n)} \rightarrow J$ such that $\left(\varphi^{(n)}\right)^{*}\left(\omega_{J}\right)=\sum_{i=1}^{n} p_{i}^{*}(\omega)$. For $n$ large enough, $\varphi^{(n)}$ is dominant, and if $\omega_{J}$ is exact, then so is $\omega$. To prove that $\omega_{J}$ is exact, we note that $J$, being a scheme of commutative groups, is uniformized by $\mathbb{C}^{n}$. We can now apply Theorem 5.4.3 of [André 2004], whose hypotheses are satisfied because the solution $f$ of the system has integer coefficients.

Alternatively, one can use a special case of a generalized Grothendieck-Katz conjecture proved by Bost, namely Corollary 2.8 in [Bost 2001, §2.4]: the vanishing of the $p$-curvatures in Condition (i) follows by a theorem of Cartier from the fact that the system has a solution in $\mathbb{F}_{p}(t)$, namely the reduction $\bmod p$ of $f$ for all prime numbers $p$ for which such a reduction of the system exists (see Exercise 3 of [André 1989, p. 84] or Theorem 5.1 of [Katz 1970]); Condition (ii) is satisfied since $\mathbb{C}^{n}$ satisfies the Liouville property. 
A nice overview of such algebraicity results is given in the Bourbaki report of Chambert-Loir [2002]; see especially Theorem 2.6 and the following lines.

\section{Examples}

Kontsevich [2011] computed $P_{\omega}$ when $\omega=X_{1}+X_{1}^{-1}+\cdots+X_{n}+X_{n}^{-1}$ considered as a $1 \times 1$ matrix, obtaining

$$
P_{\omega}=\frac{2^{n}}{(2 n-1)^{n-1}} \cdot \frac{\left(n-1+n\left(1-4(2 n-1) t^{2}\right)^{1 / 2}\right)^{n-1}}{\left(1+\left(1-4(2 n-1) t^{2}\right)^{1 / 2}\right)^{n}},
$$

which shows that $P_{\omega}$ belongs to a quadratic extension of $\mathbb{Q}(t)$.

We now present similar results for the zeta functions of two matrices: the first one of order 2 and the second one of order $d \geq 3$.

Computing $\boldsymbol{P}_{M}$ for $\boldsymbol{a} \mathbf{2} \times \mathbf{2}$ matrix. Consider the following matrix with entries in the ring $\mathbb{Z}\left\langle a, a^{-1}, b, b^{-1}, d, d^{-1}\right\rangle$, where $a, b$ and $d$ are noncommuting variables:

$$
M=\left(\begin{array}{cc}
a+a^{-1} & b \\
b^{-1} & d+d^{-1}
\end{array}\right) .
$$

Proposition 5.1. We have

$$
\begin{aligned}
& g_{M}=3 \frac{\left(1-8 t^{2}\right)^{1 / 2}-1+6 t^{2}}{1-9 t^{2}}, \\
& P_{M}=\frac{\left(1-8 t^{2}\right)^{3 / 2}-1+12 t^{2}-24 t^{4}}{32 t^{6}} .
\end{aligned}
$$

Expanding $P_{M}$ as a formal power series, we obtain

$$
P_{M}=1+\sum_{n \geq 1} \frac{3 \cdot 2^{n}}{(n+2)(n+3)}\left(\begin{array}{c}
2 n+2 \\
n+1
\end{array}\right) t^{2 n} .
$$

Proof. View the matrix $M$ under the form of the graph of Figure 1 with two vertices 1 and 2 and six labeled oriented edges. We identify paths in this graph and words on the alphabet $A=\left\{a, a^{-1}, b, b^{-1}, d, d^{-1}\right\}$. Let $B$ denote the set of nonempty words on $A$ that become trivial in the corresponding free group on $a, b$ and $d$ and whose corresponding path is a closed path. Then the integer $a_{n}(M)$ is the number of words in $B$ of length $n$. We have $\varepsilon(B)=g_{M}$, where $\varepsilon: K\langle\langle A\rangle\rangle \rightarrow K \llbracket t \rrbracket$ is the algebra map defined in Section 2.

We define $B_{i}(i=1,2)$ as the set of paths in $B$ starting from and ending at the vertex $i$; we have $B=B_{1}+B_{2}$. Each set $B_{i}$ is a free subsemigroup of $A^{*}$, freely generated by the set $C_{i}$ of closed paths not passing through $i$ (except at their ends). 


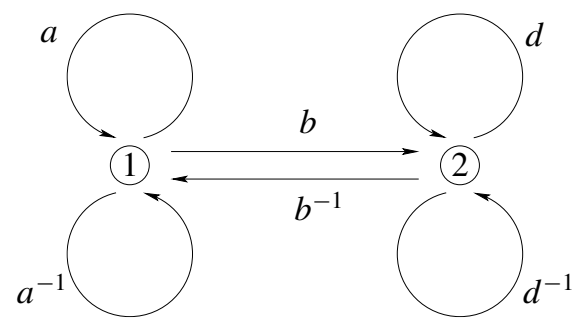

Figure 1. A graph representing $M$.

The sets $C_{i}$ do not contain the empty word. We have

$$
B_{i}=C_{i}^{+}=\sum_{n \geq 1} C_{i}^{n}, \quad i=1,2 .
$$

Given a letter $x$, we denote by $C_{i}(x)$ the set of closed paths in $C_{i}$ starting with $x$. Any word of $C_{i}(x)$ is of the form $x w x^{-1}$, where $w \in B_{j}$ when $i \stackrel{x}{\rightarrow} j$; such $w$ does not start with $x^{-1}$. Identifying a language with its characteristic series and using the standard notation $L^{*}=1+\sum_{n \geq 1} L^{n}$ for any language $L$, we obtain the equations

$$
\begin{aligned}
& C_{1}(a)=a\left(C_{1}(a)+C_{1}(b)\right)^{*} a^{-1}, \\
& C_{1}(b)=b\left(C_{2}(d)+C_{2}\left(d^{-1}\right)\right)^{*} b^{-1} .
\end{aligned}
$$

Applying the algebra map $\varepsilon$ and taking into account the symmetries of the graph, we see that the four noncommutative formal power series $C_{1}(a), C_{1}\left(a^{-1}\right), C_{2}(d)$ and $C_{2}\left(d^{-1}\right)$ are sent to the same formal power series $u \in \mathbb{Z} \llbracket t \rrbracket$ while $C_{1}(b)$ and $C_{2}\left(b^{-1}\right)$ are sent to the same formal power series $v$. It follows from (5-5) and (5-6) that $u$ and $v$ satisfy the equations

$$
u=t^{2}(u+v)^{*}=\frac{t^{2}}{1-u-v} \quad \text { and } \quad v=t^{2}(2 u)^{*}=\frac{t^{2}}{1-2 u},
$$

from which we deduce

$$
t^{2}=u(1-u-v)=v(1-2 u) .
$$

The second equality is equivalent to $(u-v)(u-1)=0$. Since $C_{1}(a)$ does not contain the empty word, the constant term of $u$ vanishes; hence, $u-1 \neq 0$. Therefore, $u=v$.

Since $C_{1}=C_{1}(a)+C_{1}\left(a^{-1}\right)+C_{1}(b)$ and $C_{2}=C_{2}(d)+C_{2}\left(d^{-1}\right)+C_{2}\left(b^{-1}\right)$, we have $\varepsilon\left(C_{1}\right)=\varepsilon\left(C_{2}\right)=2 u+v=3 u$. Therefore, $\varepsilon\left(B_{1}\right)=\varepsilon\left(B_{2}\right)=3 u /(1-3 u)$ and

$$
g_{M}=\varepsilon(B)=\frac{6 u}{1-3 u} .
$$

Let us now compute $u$ using (5-7) and the equality $u=v$. The formal power series $u$ satisfies the quadratic equation $2 u^{2}-u+t^{2}=0$. Since $u$ has zero constant term, 
we obtain

$$
u=\frac{1-\left(1-8 t^{2}\right)^{1 / 2}}{4} .
$$

From this and (5-8), we obtain the desired form for $g_{M}$.

Let $P(t)$ be the right-hand side in Equation (5-4). To prove $P_{M}=P(t)$, we checked that $t P^{\prime}(t) / P(t)=g_{M}$ and the constant term of $P(t)$ is 1 .

Remark 5.2. We found Equation (5-4) for $P(t)$ as follows. We first computed the lowest coefficients of $g_{M}$ up to degree 10 :

$$
g_{M}=6\left(t^{2}+5 t^{4}+29 t^{6}+181 t^{8}+1181 t^{10}\right)+O\left(t^{12}\right) .
$$

From this, it was not difficult to find that

$$
P_{M}=1+3 t^{2}+12 t^{4}+56 t^{6}+288 t^{8}+1584 t^{10}+O\left(t^{12}\right) .
$$

Up to a shift, the sequence (5-9) of nonzero coefficients of $P_{M}$ is the same as the sequence of numbers of "new" intervals in a Tamari lattice computed in [Chapoton 2006, §9]. (We learnt this from [OEIS 2010], where this sequence is listed as A000257.) Chapoton gave an explicit formula for the generating function $v$ of these "new" intervals (see Equation (73) in [Chapoton 2006]). Rescaling $v$, we found that $P(t)=\left(v\left(t^{2}\right)-t^{4}\right) / t^{6}$ has up to degree 10 the same expansion as (5-9). It then sufficed to check that $t P^{\prime}(t) / P(t)=g_{M}$.

By [OEIS 2010], the integers in the sequence A000257 also count the number of planar rooted bicubic maps with $2 n$ vertices (see [Tutte 1963, p. 269]). Planar maps also come up in the combinatorial interpretation of (5-1) given in [Reutenauer and Robado 2012, §5] for $n=2$.

Note that the sequence of nonzero coefficients of $g_{M} / 6$ is listed as A194723 in [OEIS 2010].

A similar $\boldsymbol{d} \times \boldsymbol{d}$ matrix. Fix an integer $d \geq 3$, and let $M$ be the $d \times d$ matrix with entries $M_{i, j}$ defined by

$$
M_{i, i}=a_{i}+a_{i}^{-1} \quad \text { and } \quad M_{i, j}= \begin{cases}b_{i j} & \text { if } i<j, \\ b_{j i}^{-1} & \text { if } j<i,\end{cases}
$$

where $a_{1}, \ldots, a_{d}, b_{i j}(1 \leq i<j \leq d)$ are noncommuting variables. This matrix is a straightforward generalization of (5-2).

Proceeding as above, we obtain two formal power series $u$ and $v$ satisfying the following equations similar to (5-7):

$$
\begin{aligned}
& u=t^{2}(u+(d-1) v)^{*}=\frac{t^{2}}{1-u-(d-1) v}, \\
& v=t^{2}(2 u+(d-2) v)^{*}=\frac{t^{2}}{1-2 u-(d-2) v} .
\end{aligned}
$$


We deduce the equality $u=v$ and the quadratic equation $u(1-d u)=t^{2}$. We finally have

$$
g_{M}=\frac{d(d+1) u}{1-(d+1) u}
$$

which leads to

$$
g_{M}=\frac{d(d+1)}{2} \frac{\left(1-4 d t^{2}\right)^{1 / 2}-1+2(d+1) t^{2}}{1-(d+1)^{2} t^{2}} .
$$

Its expansion as a formal power series is the following:

$$
\begin{aligned}
g_{M}=d(d+1)\left\{t^{2}+(2 d+1) t^{4}+\right. & \left(5 d^{2}+4 d+1\right) t^{6}+\left(14 d^{3}+14 d^{2}+6 d+1\right) t^{8} \\
& \left.+\left(42 d^{4}+48 d^{3}+27 d^{2}+8 d+1\right) t^{10}\right\}+O\left(t^{12}\right) .
\end{aligned}
$$

When $d=2,3,4$, the sequence of nonzero coefficients of $g_{M} / d(d+1)$ is listed respectively as A194723, A194724 and A194725 in [OEIS 2010] (it is also the $d$-th column in Sequence A183134). These sequences count the $d$-ary words, either empty or beginning with the first letter of the alphabet, that can be built by inserting $n$ doublets into the initially empty word.

We were not able to find a closed formula for $P_{M}$ analogous to (5-4). Using Maple, we found that, for instance up to degree 10, the expansion of $P_{M}$ is

$$
\begin{aligned}
1+\frac{d(d+1)}{2} t^{2} & +\frac{d(d+1)\left(d^{2}+5 d+2\right)}{8} t^{4} \\
& +\frac{d(d+1)\left(d^{4}+14 d^{3}+59 d^{2}+38 d+8\right)}{48} t^{6} \\
& +\frac{d(d+1)\left(d^{6}+27 d^{5}+271 d^{4}+1105 d^{3}+904 d^{2}+332 d+48\right)}{384} t^{8} .
\end{aligned}
$$

\section{Acknowledgements}

We are most grateful to Yves André, Jean-Benoît Bost and Carlo Gasbarri for their help in the proof of Theorem 4.4. We are also indebted to François Bergeron and Pierre Guillot for assisting us with computer computations in the process detailed in Remark 5.2, to Frédéric Chapoton for his comments on the last part of Section 5 and to an anonymous referee for having spotted slight inaccuracies. Thanks also to Stavros Garoufalidis for pointing out references [Garoufalidis and Bellissard 2007; Sauer 2003], in which the algebraicity of $g_{M}$ had been proved.

Kassel was partially funded by the Laboratoire International Franco-Québécois de Recherche en Combinatoire (LIRCO) and Université du Québec à Montréal (UQAM). Reutenauer is supported by NSERC (Canada). 


\section{References}

[André 1989] Y. André, G-functions and geometry, Aspects of Mathematics 13, Friedr. Vieweg \& Sohn, Braunschweig, 1989. MR 90k:11087 Zbl 0688.10032

[André 2004] Y. André, "Sur la conjecture des p-courbures de Grothendieck-Katz et un problème de Dwork", pp. 55-112 in Geometric aspects of Dwork theory, vol. I, edited by A. Adolphson et al., Walter de Gruyter GmbH \& Co. KG, Berlin, 2004. MR 2006d:12005 Zbl 1102.12004

[Berstel 1979] J. Berstel, Transductions and context-free languages, Leitfäden der Angewandten Mathematik und Mechanik 38, B. G. Teubner, Stuttgart, 1979. MR 80j:68056 Zbl 0424.68040

[Berstel and Reutenauer 1990] J. Berstel and C. Reutenauer, "Zeta functions of formal languages", Trans. Amer. Math. Soc. 321:2 (1990), 533-546. MR 91f:68110 Zbl 0797.68092

[Berstel and Reutenauer 2011] J. Berstel and C. Reutenauer, Noncommutative rational series with applications, Encyclopedia of Mathematics and its Applications 137, Cambridge University Press, 2011. MR 2012b:68152 Zbl 1250.68007

[Bost 2001] J.-B. Bost, "Algebraic leaves of algebraic foliations over number fields", Publ. Math. Inst. Hautes Études Sci. 93 (2001), 161-221. MR 2002h:14037 Zbl 1034.14010

[Bousquet-Mélou 2005] M. Bousquet-Mélou, "Algebraic generating functions in enumerative combinatorics and context-free languages", pp. 18-35 in STACS 2005, edited by V. Diekert and B. Durand, Lecture Notes in Comput. Sci. 3404, Springer, Berlin, 2005. MR 2006c:05012 Zbl 1118.05300

[Chambert-Loir 2002] A. Chambert-Loir, “Théorèmes d'algébricité en géométrie diophantienne (d'après J.-B. Bost, Y. André, D. \& G. Chudnovsky)”, pp. 175-209 in Séminaire Bourbaki 2000/2001, Astérisque 282, Société Mathématique de France, Paris, 2002. MR 2004f:11062 Zbl 1044.11055

[Chapoton 2006] F. Chapoton, "Sur le nombre d'intervalles dans les treillis de Tamari”, Sém. Lothar. Combin. 55 (2006), Art. B55f. MR 2007g:05009 Zbl 1207.05011

[Chomsky and Schützenberger 1963] N. Chomsky and M. P. Schützenberger, "The algebraic theory of context-free languages", pp. 118-161 in Computer programming and formal systems, edited by P. Braffort and D. Hirschberg, North-Holland, Amsterdam, 1963. MR 27 \#2371 Zbl 0148.00804

[Chudnovsky and Chudnovsky 1985] D. V. Chudnovsky and G. V. Chudnovsky, "Applications of Padé approximations to the Grothendieck conjecture on linear differential equations", pp. 52-100 in Number theory (New York, 1983-1984), edited by D. V. Chudnovsky et al., Lecture Notes in Math. 1135, Springer, Berlin, 1985. MR 87d:11053 Zbl 0565.14010

[Garoufalidis and Bellissard 2007] S. Garoufalidis and J. Bellissard, "Algebraic $G$-functions associated to matrices over a group-ring”, preprint, 2007. arXiv 0708.4234v4

[Jacob 1975] G. Jacob, "Sur un théorème de Shamir", Information and Control 27 (1975), 218-261. MR 51 \#2361 Zbl 0318.68053

[Katz 1970] N. M. Katz, "Nilpotent connections and the monodromy theorem: applications of a result of Turrittin”, Inst. Hautes Études Sci. Publ. Math. 39 (1970), 175-232. MR 45 \#271 Zbl 0221.14007

[Kontsevich 2011] M. Kontsevich, "Noncommutative identities", notes of talk at Mathematische Arbeitstagung, Bonn, 2011. arXiv 1109.2469v1

[OEIS 2010] "The on-line encyclopedia of integer sequences", 2010, http://oeis.org.

[Reutenauer and Robado 2012] C. Reutenauer and M. Robado, "On an algebraicity theorem of Kontsevich”, pp. 239-246 in 24th International Conference on Formal Power Series and Algebraic Combinatorics (Nagoya, 2012), Assoc. Discrete Math. Theor. Comput. Sci., Nancy, 2012. MR 2958001

[Salomaa and Soittola 1978] A. Salomaa and M. Soittola, Automata-theoretic aspects of formal power series, Springer, New York, 1978. MR 58 \#3698 Zbl 0377.68039 
Algebraicity of zeta functions associated to a matrix over a free group algebra 511

[Sauer 2003] R. Sauer, "Power series over the group ring of a free group and applications to NovikovShubin invariants", pp. 449-468 in High-dimensional manifold topology, edited by F. T. Farrell and W. Lück, World Sci. Publ., 2003. MR 2005b:16078 Zbl 1051.16013

[Schützenberger 1962] M. P. Schützenberger, “On a theorem of R. Jungen”, Proc. Amer. Math. Soc. 13 (1962), 885-890. MR 26 \#350 Zbl 0107.03102

[Stanley 1999] R. P. Stanley, Enumerative combinatorics, vol. 2, Cambridge Studies in Advanced Mathematics 62, Cambridge University Press, 1999. MR 2000k:05026 Zbl 0928.05001

[Tutte 1963] W. T. Tutte, “A census of planar maps", Canad. J. Math. 15 (1963), 249-271. MR 26 \#4343 Zbl 0115.17305

Communicated by Victor Reiner

Received 2013-04-25 Revised 2013-07-15 Accepted 2013-07-24

kassel@math.unistra.fr Institut de Recherche Mathématique Avancée, CNRS, Université de Strasbourg, 7 rue René Descartes, 67084 Strasbourg, France

reutenauer.christophe@uqam.ca Mathématiques, Université du Québec à Montréal, CP 8888 succursale Centre Ville, Montréal QC H3C 3P8, Canada 


\section{Algebra \& Number Theory}

msp.org/ant

\section{EDITORS}

MANAGING EDITOR

Bjorn Poonen

Massachusetts Institute of Technology

Cambridge, USA

\author{
EDITORIAL BOARD CHAIR \\ David Eisenbud \\ University of California \\ Berkeley, USA
}

\section{BOARD OF EDITORS}

Georgia Benkart

Dave Benson

Richard E. Borcherds

John H. Coates

J-L. Colliot-Thélène

Brian D. Conrad

Hélène Esnault

Hubert Flenner

Edward Frenkel

Andrew Granville

Joseph Gubeladze

Roger Heath-Brown

Ehud Hrushovski

Craig Huneke

Mikhail Kapranov

Yujiro Kawamata

János Kollár

Yuri Manin

Barry Mazur

Philippe Michel
University of Wisconsin, Madison, USA

University of Aberdeen, Scotland

University of California, Berkeley, USA

University of Cambridge, UK

CNRS, Université Paris-Sud, France

University of Michigan, USA

Freie Universität Berlin, Germany

Ruhr-Universität, Germany

University of California, Berkeley, USA

Université de Montréal, Canada

San Francisco State University, USA

Oxford University, UK

Hebrew University, Israel

University of Virginia, USA

Yale University, USA

University of Tokyo, Japan

Princeton University, USA

Northwestern University, USA

Harvard University, USA

École Polytechnique Fédérale de Lausanne
Susan Montgomery

Shigefumi Mori

Raman Parimala

Jonathan Pila

Victor Reiner

Karl Rubin

Peter Sarnak

Joseph H. Silverman

Michael Singer

Vasudevan Srinivas

J. Toby Stafford

Bernd Sturmfels

Richard Taylor

Ravi Vakil

Michel van den Bergh

Marie-France Vignéras

Kei-Ichi Watanabe

Efim Zelmanov

Shou-Wu Zhang
University of Southern California, USA

RIMS, Kyoto University, Japan

Emory University, USA

University of Oxford, UK

University of Minnesota, USA

University of California, Irvine, USA

Princeton University, USA

Brown University, USA

North Carolina State University, USA

Tata Inst. of Fund. Research, India

University of Michigan, USA

University of California, Berkeley, USA

Harvard University, USA

Stanford University, USA

Hasselt University, Belgium

Université Paris VII, France

Nihon University, Japan

University of California, San Diego, USA

Princeton University, USA

PRODUCTION

production@msp.org

Silvio Levy, Scientific Editor

See inside back cover or msp.org/ant for submission instructions.

The subscription price for 2014 is US $\$ 225 /$ year for the electronic version, and $\$ 400 /$ year $(+\$ 55$, if shipping outside the US) for print and electronic. Subscriptions, requests for back issues and changes of subscribers address should be sent to MSP.

Algebra \& Number Theory (ISSN 1944-7833 electronic, 1937-0652 printed) at Mathematical Sciences Publishers, 798 Evans Hall \#3840, c/o University of California, Berkeley, CA 94720-3840 is published continuously online. Periodical rate postage paid at Berkeley, CA 94704, and additional mailing offices.

ANT peer review and production are managed by EditFLOW ${ }^{\circledR}$ from Mathematical Sciences Publishers.

\section{PUBLISHED BY}

- mathematical sciences publishers

nonprofit scientific publishing

http://msp.org/

(C) 2014 Mathematical Sciences Publishers 


\section{Algebra \& Number Theory}

Volume $8 \quad$ No. $2 \quad 2014$

Large self-injective rings and the generating hypothesis

LEIGH SHEPPERSON and NEIL STRICKLAND

On lower ramification subgroups and canonical subgroups

303

SHIN HATTORI

Wild models of curves

DINO LORENZINI

Geometry of Wachspress surfaces

COREY IRVING and HAL SCHENCK

Daniel Goldstein, Robert M. GuRAlnick, Mark L. Lewis, AleXander Moretó, Gabriel Navarro and Pham HuU TieP

The homotopy category of injectives

AMNON NEEMAN

Essential dimension of spinor and Clifford groups

Vladimir Chernousov and AleXANDER MERKurJeV

On Deligne's category $\underline{\operatorname{Rep}}^{a b}\left(S_{d}\right)$

JONATHAN COMES and VICTOR OSTRIK

Algebraicity of the zeta function associated to a matrix over a free group algebra

CHRISTIAN KASSEL and Christophe REUTENAUER 